\title{
Identificación de Parásitos Gastrointestinales por Coproscopía en Carnívoros Silvestres del Zoológico Parque de las Leyendas, Lima, Perú
}

\author{
Identification of Gastrointestinal Parasites by Coproscopy in Wild Carnivores \\ from the Parque de las Leyendas, Lima, Peru
}

Maritza Acosta Z. ${ }^{1,3}$, Manuel Tantaleán V. ${ }^{2}$, Enrique Serrano-Martínez ${ }^{1,4}$

\section{Resumen}

\begin{abstract}
El presente trabajo tuvo por objetivo identificar los parásitos gastrointestinales de carnívoros silvestres del Zoológico Parque de Las Leyendas en el Perú, mediante la aplicación de cuatro métodos coproparasitológicos convencionales (directo, de Ritchie modificado, Sheather y la coloración de Ziehl Neelsen). Se trabajó con 62 ejemplares pertenecientes a 17 especies de seis familias. El 25.8\% (16/62) de las muestras fueron positivas a parásitos. Panthera leo, Panthera tigris y Lycalopex sechurae fueron las especies más parasitadas $(9 / 9,2 / 3$ y $3 / 5$, respectivamente). Los parásitos identificados fueron el nematodo Toxascaris leonina (12/16), el acantocéfalo Corynosoma sp (3/16), el cestodo Atriotaenia sp (1/6) y el protozoo Giardia sp. (1/16). No se encontró asociación estadística entre las variables de edad y sexo.
\end{abstract}

Palabras clave: parásitos gastrointestinales, carnívoros silvestres, Parque de Las Leyendas, Perú

\section{Abstract}

The aim of this study was to identify gastrointestinal parasites in wild carnivores from the Parque de Las Leyendas Zoo in Peru by four conventional coproparasitological methods (direct, Ritchie modified Sheather and the Ziehl Neelsen method for coccidia). A total of 62 animals of 15 species from six families were sampled. Results showed that

\footnotetext{
${ }^{1}$ Laboratorio de Parasitología, Facultad de Medicina Veterinaria y Zootecnia, Universidad Peruana Cayetano Heredia, Lima, Perú

${ }^{2}$ Laboratorio de Parasitología en Fauna Silvestre y Zoonosis, Facultad de Ciencias Biológicas, Universidad Nacional Mayor de San Marcos, Lima, Perú

${ }^{3}$ E-mail: maritza.acosta@upch.pe

${ }^{4}$ E-mail:marcos.serrano@upch.pe
}

Recibido: 18 de setiembre de 2014

Aceptado para publicación: 24 de diciembre de 2014 
$25.8 \%$ (16/62) of the samples were positive to parasites. Panthera leo, Panthera tigris and Lycalopex sechurae were the most affected species (9/9, 2/3, and $3 / 5$ respectively). The parasites identified were the nematode Toxascaris leonina (12/16), the acanthocephalan Corynosoma sp (3/16), the cestode Atriotaenia sp (1/6) and the protozoo Giardia sp (1/6). No significant association was found between the variables age and sex.

Key words: gastrointestinal parasites, wild carnivores, Parque de Las Leyendas, Peru

\section{INTRODUCCIÓN}

Entre los carnívoros silvestres en cautiverio en el Perú se encuentran el gato de pajonal (Leopardus colocolo), tigrillo u ocelote (Leopardus pardalis), oncilla (Leopardus tigrinus), margay (Leopardus wiedii), puma (Puma concolor) y jaguar u otorongo (Panthera onca), pertenecientes a la familia Felidae (Olivieira et al., 2001). Asimismo, el zorro andino (Lycalopex culpaeus) y zorro costeño (Lycalopex sechurae) de la familia Canidae (Pessutti et al., 2001), la chosna (Potos flavus) y coatí (Nasua nasua) de la familia Procyonidae (Sampaio et al., 2001), la nutria de río (Lontra longicaudis) de la familia Mustelidae (Lucena et al., 2001) y el lobo chusco (Otaria flavescens) de la familia Otariidae (Pacheco et al., 2009).

Una de las importantes funciones ecológicas que desempeñan los carnívoros silvestres es la de regular las poblaciones de especies presa. Sin embargo, constituyen uno de los grupos menos estudiados y son víctimas de muchas formas de amenaza; entre ellas la fragmentación del hábitat, la caza para el comercio de mascotas o de sus pieles, la caza furtiva y deportiva, y la eliminación de individuos problema en resguardo del ganado y otros animales domésticos. A esto, se agrega el tráfico ilegal, nacional e internacional, de especies silvestres vivas o de sus productos derivados (Pacheco et al., 2009).

Debido a estas amenazas que afectan sus poblaciones, la Convención sobre el Co- mercio Internacional de Especies Amenazadas de Fauna y Flora Silvestres ha registrado el estado de conservación de cada una de estas especies, ubicándose los géneros Tremarctos, Ursus, Leopardus, Panthera, Puma y Lontra en el apéndice I y Lycalopex en el apéndice II; mientras que Nasua y Potos se encuentran en el apéndice III (CITES, 2009). La amenaza que se cierne sobre estas especies para su supervivencia, requiere en muchos casos la necesidad de su crianza en cautiverio para contribuir con su conservación.

Los carnívoros silvestres son reservorios de una gran variedad de parásitos, algunos de los cuales pueden permanecer en el hospedero en condiciones de cautiverio debido a la autoinfección o reinfección, cuando las condiciones sanitarias, alimento y presencia de hospederos intermediarios o vectores lo permiten (Arrojo, 2002; Tantal eán y Michaud, 2005). Asimismo, dependiendo de la especie, localización y condiciones devida del animal, la patogenicidad de los parásitos puede exacerbarse y ocasionar la muerte del hospedero (Arrojo, 2002). El cautiverio es uno delos factores que induce a estrés, disminuyendo la capacidad inmunológica del animal y propiciando el surgimiento de las parasitosis (Müller et al., 2005).

Estudios previos relacionados con la presencia de parásitos en varias especies de animales en fauna silvestre en el Perú se han realizado en félidos (Aranda, 2013), primates (Guerrero et al., 2012), reptiles (Chávez, 2011) y en el venado de cola blanca 
(Odocoileus virginianus) (Céspedes, 2011); asimismo, estudios realizados en el zoológico Parque de Las Leyendas de Lima, revelaron la presencia de huevos de Spirometra mansonoides en pumas, otorongos y tigrillos (Tantaleán y Michaud, 2005). Estos hallazgos permitieron establecer medidas de control y planes de tratamiento adecuados; sin embargo, no se realizaron nuevos estudios en estas especies por lo que se hace indispensable seguir investigando. Por todas estas razones, el presente estudio se diseñó a fin de determinar si las especies de carnívoros criados en cautiverio en este zoológico son portadores de parásitos gastrointestinales.

\section{Materiales y Métodos}

Se trabajó con los carnívoros silvestres criados en cautiverio en el zoológico Parque de las Leyendas, localizado en el distrito de San Miguel, Lima, Perú. Los ambientes de los animales, con excepción de los coatíes, contaban con dormideros cercados por paredes y techo de concreto, puertas y rejas de fierro, y pisos de cemento pulido aseados diariamente con agua.

El manejo sanitario de estas especies se realizaba una vez al año, el cual consiste en el pesaje, recorte de uñas o garras, limpieza de dientes y orejas, y la aplicación de vitaminas, vacunas y antiparasitarios. La frecuencia del uso de antiparasitarios era dependiente de la observación de parásitos en las heces o a través de exámenes coproparasitológicos. Estos últimos se practican en forma esporádica, especialmente cuando los animales muestran signos clínicos de enfermedad.

Entre diciembre de 2012 y enero de 2013, se recolectaron muestras de heces de 62 carnívoros silvestres, distribuidos en 17 especies de seis familias (Cuadro 1). Los animales se agruparon de acuerdo a la edad (jóvenes [ $\mathrm{n}=23]$, adultos [ $\mathrm{n}=39]$ ) y sexo (machos $[n=29]$, hembras $[n=33]$ ).
El criterio de inclusión considerado fue que los animales no hayan sido tratados con antiparasitarios en los tres meses previos al estudio y que las muestras de heces estuvieran frescas y bien preservadas. Como criterio de exclusión se consideraron a los carnívoros en calidad de pacientes del hospital veterinario y aquellos en cuarentena.

Previamente a la colección de heces, se realizó una inspección general de los animales para observar su estado de salud, condición corporal y consumo de alimento; además, se revisaron las historias clínicas para buscar antecedentes de parasitosis. Los animales provenían de decomiso, abandono, custodia, nacidos en cautiverio o fueron donados por otras instituciones.

Las muestras se recogieron del suelo, tomando una por animal. En el caso de animales alojados en grupo, las muestras se obtuvieron al momento de defecar. En caso de no ser posible, se procedió a recoger todas las muestras que se encontraban en el dormidero. Las muestras fueron rotuladas y mantenidas en refrigeración a $4{ }^{\circ} \mathrm{C}$. Una parte de cada muestra se fijó con formol al $10 \%$ calentado a $65{ }^{\circ} \mathrm{C}$ y la otra parte, siempre que tuviera ooquistes (observados en el microscopio), se acondicionaron para favorecer la esporulación. Asimismo, se realizó un frotis de la muestra fresca para la coloración de Ziehl-Neelsen para el estudio de Cryptosporidium.

Las muestras de heces fueron procesadas en el Laboratorio de Parasitología de la Facultad de Medicina Veterinaria y Zootecnia, Universidad Peruana Cayetano Heredia (UPCH), Lima, Perú. Todas las muestras fueron procesadas mediante cuatro técnicas para la búsqueda de huevos de helmintos, coccidias y protozoos: método directo, de Ritchie (sedimentación), de Sheather (Flotación) y la coloración de ZiehlNeelsen para coccidios. Los resultados de frecuencia se expresan en forma porcentual (Thrusfield, 1990) y la asociación entre variables fue analizada mediante la prueba de Fisher exacto. 


\section{Resultados}

Se encontraron cuatro géneros de parásitos gastrointestinales en cinco especies de hospederos (Cuadro 2, Fig. 1). No se detectó Cryptosporidium ni otro tipo de protozoario.

Del total de 62 muestras, 16 resultaron positivas a alguna forma de parásito, representando un $25.8 \%$ (16/62), siendo el león (Panthera leo), la especie más parasitada con $100 \%$ de frecuencia. El parásito con la más alta frecuencia fue Toxascaris leoni$n a$, al estar presente en 12 de los 16 animales estudiados $(75 \%)$, seguida por Corynosoma (18.75\%, 3/16). Asimismo, es interesante indicar que en una muestra de un tigre (Panthera tigris) se encontraron huevos y quistes de dos especies de parásitos ( $T$. leonina y Giardia sp).

El Cuadro 3 muestra la frecuencia de parasitosis gastrointestinal según edad y sexo. El 39.1\% (9/23) de los animales jóvenes y el $17.9 \%(7 / 39)$ de los adultos estaban parasitados. Asimismo, el 37.9\% (11/29) de los machos y el $15.2 \%(5 / 33)$ de las hembras estaban parasitados.

\section{Discusión}

La mayoría de infecciones parasitarias gastrointestinales son asintomáticas, afectando mayormente a los animales jóvenes (Müller et al., 2005). La carga parasitaria disminuye la coloración del pelaje y afecta el consumo de alimentos (Suzán et al., 2000). Las posibles fuentes de infección en los zoológicos son roedores y pájaros silvestres que tienen acceso a las zonas que ocupan los animales, así como por residuos en el calzado de los manipuladores. Además, las modificaciones ambientales favorecen la diseminación de enfermedades, particularmente aquellas transmitidas por vectores (Müller et al., 2005).
La frecuencia encontrada de parasitosis gastrointestinal fue de $25.8 \%$; cifra relativamente baja en comparación con el 95 y $61 \%$ encontrados en zoológicos de Brasil (Figueiroa et al., 2001; Müller et al., 2005) y el $62 \%$ en otros zoológicos del Perú (Aranda, 2013). Asimismo, Beltrán-Saavedra et al. (2009) reportan $66.7 \%$ de animales infectados en un centro de rescate de Bolivia. Esta diferencia podría deberse a las mejores condiciones de manejo (ambiental y sanitario), incluyendo la limitación de las fuentes de infección, en que se encuentran los animales del Parque de las Leyendas (Suzán et al., 2000; Müller et al., 2005). Por otro lado, la frecuencia de parasitismo hallada es menor que la observada en estudios realizados en vida libre, donde hay mayores fuentes de infección y presencia de hospederos intermediarios (Patton y Rabinowitz, 1994; Figueiroa et al., 2001; Tantaleán y Michaud, 2005; Fiorello et al., 2006).

Los parásitos hallados en los diferentes hospederos coinciden con estudios previos en carnívoros silvestres de vida libre y en cautiverio. El tipo de dormidero de los animales y el aseo diario con agua pueden generar condiciones de humedad, temperatura y oxígeno adecuadas para la presencia de Toxascaris sp y Giardia sp; asimismo, el sustrato de los ambientes de exhibición es de tierra húmeda con pasto, arbustos y árboles que son regados diariamente, el cual crea un ambiente propicio para la continuación del ciclo biológico de Atriotaenia que requiere como hospedero intermediario a un ácaro.

Toxascaris leonina ha sido reportado en L. pardalis de vida libre en Texas (Pence et al., 2003) y en pumas de La Florida, EEUU (Dunbar, 1994), en otorongos en América Central (Patton et al., 1986) y en carnívoros silvestres (L. pardalis, P. onca, P. concolor) de Brasil (Vieira et al., 2008). Asimismo, ha sido reportado en $P$. onca y $L$. wiedii silvestres en el Perú (Aranda, 2013). 
Cuadro 1. Carnívoros silvestres criados en cautiverio en el Zoológico Parque de Las Leyendas (Lima, diciembre 2012 - enero 2013)

\begin{tabular}{lllcc}
\hline \multirow{2}{*}{ Familia } & Especie & Nombre común & \multicolumn{2}{c}{$\mathrm{N}^{\circ}$ de individuos } \\
\cline { 3 - 5 } Canidae & Lycalopex culpaeus & Zorro andino & 2 & 1 \\
& Lycalopex sechurae & Zorro costeño & 4 & 1 \\
\multirow{2}{*}{ Ursidae } & Tremarctos ornatus & Oso de anteojos & 3 & 4 \\
& Ursus arctos arctos & Oso pardo & 1 & 1 \\
& Ursus americanus & Oso negro americano & 1 & 1 \\
Procyonidae & Nasua nasua & Coatí & 1 & 1 \\
& Potos flavus & Chosna & 1 & 7 \\
Fustelidae & Lontra longicaudis & Nutria de río & - & 1 \\
& Leopardus pardalis & Tigrillo & 2 & - \\
& Leopardus tigrinus & Oncilla & 1 & 2 \\
& Leopardus wiedii & Margay & 2 & - \\
& Leopardus colocolo & Gato del pajonal & 1 & 1 \\
& Panthera onca & Otorongo & 1 & 4 \\
& Puma concolor & Puma & 2 & 1 \\
& Panthera leo & León & 6 & 3 \\
& Panthera tigris & Tigre & 1 & 2 \\
& Otariaflavescens & Lobo chusco & 1 & 2 \\
\hline
\end{tabular}

Cuadro 2. Parásitos gastrointestinales encontrados en carnívoros silvestres en cautiverio en el Zoológico Parque de Las Leyendas (Lima, diciembre 2012-enero 2013)

\begin{tabular}{llcc}
\hline Parásito & Hospedero & $\begin{array}{c}\text { Muestras } \\
(\mathrm{n})\end{array}$ & $\begin{array}{c}\text { Muestras positivas } \\
(\%)\end{array}$ \\
\hline Toxascaris leonina & Panthera leo & 9 & 100.0 \\
& Puma concolor & 3 & 33.3 \\
& Panthera tigris & 3 & 66.7 \\
Giardia $\mathrm{sp}$ & Panthera tigris & 3 & 33.3 \\
Corynosoma $\mathrm{sp}$ & Lycalopex sechurae & 5 & 60.0 \\
Atriotaenia $\mathrm{sp}$ & Nasua nasua & 2 & 50.0 \\
\hline
\end{tabular}



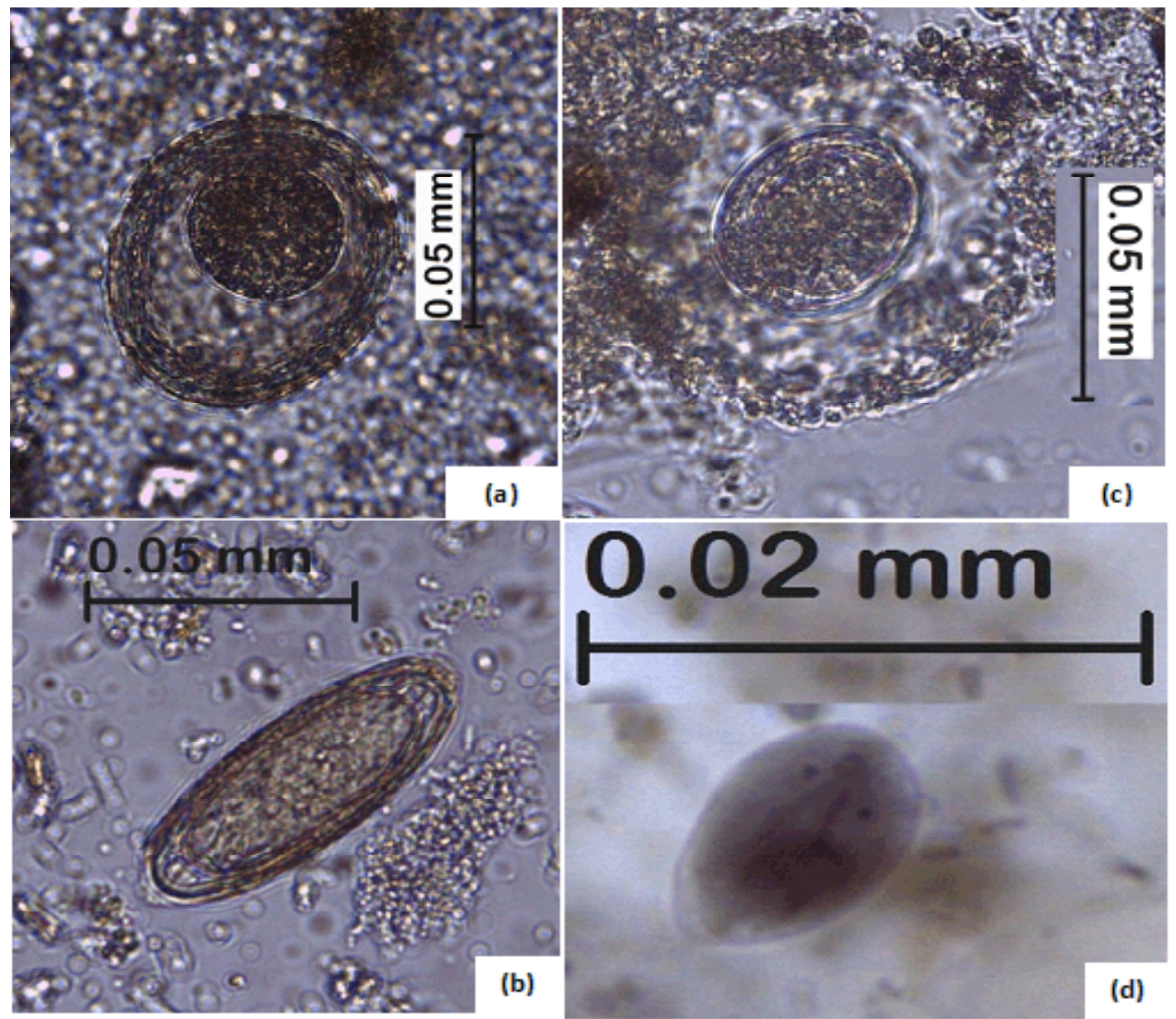

Figura 1. Huevos y quistes de parásitos gastrointestinales de carnívoros silvestres en cautiverio del Zoológico Parque de Las Leyendas (Lima, diciembre 2012 - enero 2013). (a) Huevo de Toxascaris leonina; (b) Huevo de Corynosoma sp; (c) Huevo de Atriotaenia sp; (d) Quiste de Giardia sp

Cuadro 3. Frecuencia de parasitosis gastrointestinales según edad y sexo en carnívoros silvestres en cautiverio en el Zoológico Parque de Las Leyendas (diciembre 2012enero 2013)

\begin{tabular}{lllc}
\hline \multirow{2}{*}{ Variable } & & $\begin{array}{c}\text { Animales muestreados } \\
(\mathrm{n})\end{array}$ & $\begin{array}{c}\text { Animales positivos } \\
(\%)\end{array}$ \\
\hline \multirow{2}{*}{ Edad } & Jóvenes & 23 & 39.1 \\
& Adultos & 39 & 17.9 \\
\cline { 2 - 4 } & Total & 62 & 25.8 \\
\hline \multirow{2}{*}{ Sexo } & Macho & 29 & 37.9 \\
& Hembra & 33 & 15.2 \\
& Total & 62 & 25.8 \\
\hline
\end{tabular}


En el presente estudio solo se identificaron quistes de Giardia sp en P. tigris. Este protozoo ha sido reportado en $L$. wiedii, $L$. tigrinus, $P$. concolor y $P$. onca en zoológicos de Brasil (Müller et al., 2005; Belmonte et al., 2008) y en $P$. concolor y $L$. wiedii en cautiverio en el Perú (Aranda, 2013).

La presencia de acantocéfalos como Oncicola sp en cánidos silvestres ha sido mencionada por varios autores (Patton y Rabinowitz, 1994; Pence et al., 2003; Tantaleán et al., 2005; Vieira et al., 2008; Aranda, 2013); sin embargo, en el presente estudio solo se encontraron huevos de Corynosoma sp, una especie que infecta a lobos marinos y en cuyo ciclo biológico intervienen como hospedero paraténico numerosas especies de peces marinos carnívoros (Tantaleán y Huiza, 1994; Castro y Martínez, 2004). Este parásito ha sido encontrado en el $100 \%$ de la población de lobos marinos, frente a las costas de La Libertad e Ica (Miranda et al., 1968). Asimismo, los estadios infectantes se encuentran en la mayoría de las especies de peces de la costa peruana (Tantaleán et al., 2007). Este hallazgo es importante porque supone la ingesta de peces marinos como parte de la dieta alimenticia; no obstante, este parásito también ha sido reportado en el zorro Pseudalopex culpaeus como hospedero definitivo accidental (Tantaleán et al., 2007) y en el perro doméstico (Cabrera et al., 1999).

Especies de Atriotaenia han sido reportadas por Gómez-Puerta et al. (2012) en carnívoros silvestres. Así, A. sandgroundi en Nasua nasua (coatí), A. procyonis en Procyon lotor (mapache boreal), A. incisa en Meles meles (tejón común). Asimismo, estos autores describieron una nueva especie, A. sanmarci, en Conepatus chinga (zorrillo andino) en Cusco, Perú, siendo la quinta especie conocida de Atriotaenia. En el presente estudio no fue posible la identificación de la especie; sin embargo, este hallazgo coincide con lo señalado por Vieira et al. (2008), quienes también describen su presencia en $N$. nasua en Brasil.
T. leonina fue el parásito más observado $(75 \%)$, especialmente en leones, lo cual coincide con los hallazgos de Müller-Graf (1995), quien señala que Toxascaris es comúnmente observado en leones de zoológicos en África. Por otra parte, se le ha encontrado en $100 \%$ de $L$. pardalis de vida libre en Texas, EEUU (Pence et al., 2003). La presencia de este parásito en felinos en cautiverio se debería a la ingestión de huevos infectantes presentes en el piso del alojamiento o por la ingestión de la larva infectante enquistada en los tejidos de hospederos paraténicos (Pence et al., 2003).

La frecuencia de Giardia sp fue baja, ya que solo un ejemplar de $P$. tigris estuvo infectado. Müller et al. (2005) observaron una infección cerca de $40 \%$ en félidos de dos zoológicos en Brasil, en tanto que Belmonte et al. (2008) identificó a este parásito en un ejemplar de $L$. wiedii de vida libre en Brasil. Asimismo, Aranda (2013) lo encontró en 11\% de félidos en el Perú. La baja frecuencia estaría asociada al alojamiento de estos animales en pisos de concreto, limpiados diariamente, lo cual disminuiría la carga parasitaria (Martínez et al., 2002).

\section{Conclusiones}

- Se hallaron tres especies de helmintos (Toxascaris leonina, Corynosoma sp y Atriotaenia sp) y una especie de protozoarios (Giardia sp), donde la mayor frecuencia de presentación fue de T. leonina.

- Los hallazgos de Corynosoma sp en Lycalopex sechurae y de Atriotaenia sp en Nasua nasua en cautiverio constituyen los primeros reportes en el Perú.

\section{Agradecimientos}

Los autores agradecen al personal profesional y técnico del Zoológico Parque de Las Leyendas, por su apoyo durante la colección de muestras y desarrollo del presente estudio. 


\section{Literatura Citada}

1. Aranda C. 2013. Identificación y frecuencia de parásitos gastrointestinales en félidos silvestres en cautiverio en el Perú. Rev Inv Vet Perú 24: 360-368.

2. Arrojo L. 2002. Parásitos de animales silvestres en cautiverio en Lima, Perú. Rev Perú Biol 9: 118-120.

3. Belmonte C, Soares JF, Schafer da Silva A, Kipper da Silva M, Salomão EL, Gonzales S. 2008. Ocorrência de Giardia sp e Cryptosporidium sp em Leopardus weidii de vida libre. Cienc Rural 38: 546-547.

4. Beltrán-Saavedra LF, Beldoménico PM, Gonzáles JL. 2009. Estudio coproparasitológico de mamíferos silvestres en cautiverio con destino a relocación en Santa Cruz, Bolivia. Vet Zootec 3: 51-60.

5. Cabrera $R$, Rojas $R$, Dávalos $M$. 1999. Corynosoma obtuscens Lincicome, 1943 (Acanthocephala, Polymorphidae) en Canis familiaris de la ciudad de Chincha, Perú. Parasitol Dia 23: 58-61.

6. Castro M, Martínez R. 2004. Proceso del desarrollo de Corynosoma obtuscens (Acanthocephala, Polimorphidae) en Canis familiaris y su posible implicancia en salud pública. Parasitol Latinoam 59: 26-30. doi: 10.4067/S071777122004000100005

7. Céspedes S. 2011. Identificación de parásitos sanguíneos y gastrointestinales del venado de cola blanca (Odocoileus virginianus) mantenido en cautiverio en el Zoológico Parque de Las Leyendas, Lima, Perú. Tesis de Médico Veterinario Zootecnista. Lima: Univ Peruana Cayetano Heredia. 28 p.

8. Chávez L. 2011. Identificación de parásitos gastrointestinales en reptiles mantenidos en cautiverio de Lima Metropolitana. Tesis de Médico Veterinario Zootecnista. Lima: Univ Peruana Cayetano Heredia. 27 p.
9. [CITES] Convención sobre el Comercio Internacional de Especies Amenazadas de Fauna y Flora Silvestre. 2009. Apéndices I, II y III. Ginebra: CITES. $41 \mathrm{p}$.

10. Dunbar MR. 1994. Florida panther biomedical investigations. In: Florida Panther Conference. EEUU. p 343-394.

11. Figueiroa M, Bianque J, Dowell M, Alves R. Evencio A. 2001. Perfil coproparasitológico de mamíferos silvestres en cautiverio en el estado de Pernambuco, Brasil. Parasitol Día 25: 34. doi: 10.4067/S0716-07202001000300009

12. Fiorello CV, Robbins RG, Maffei L, Wade SE. 2006. Parasites of freeranging small canids and felids in the Bolivian Chaco. J Zoo Wildl Med 37: 130-134.

13. Gómez-Puerta LA, Ticona DS, LópezUrbina MT, González AE. 2012. A new species of Atriotaenia (Cestoda: Anoplocephalidae) from the hog-nosed skunk Conepatus chinga (Carnivora: Mephitidae) in Peru. J Parasitol 98: 806809. doi: 10.1645/GE-2872.1

14. González-Acuña, D. Moreno, L. Ardiles, K. Flores, M. Duclos, M. Kinsella, M. 2010. Endoparasites of the kodkod, Oncifelis guigna (Carnivora, Felidae) in Chile. Rev Chil Hist Nat 83: 619-622. doi: 10.4067/S0716-078X2010000400015

15. Guerrero F, Serrano E, Tantaleán M, Quispe M, Casas G. 2012. Identificación de parásitos gastrointestinales en primates no humanos del zoológico parque natural de Pucallpa, Perú. Ver Inv Vet Perú 23: 469-478.

16. Lucena T, Lima M, Passerino A. 2001. Order Carnivora, Family Mustelidae. In: Fowler ME, Cubas Z (eds). Biology, medicine, and surgery of South American wild animals. EEUU: Wiley-Blackwell. p 323-331.

17. Martínez FA, Troiano JC, Gauna L, Rearte R, Jara D. 2002. Infección por coccidios en carnívoros silvestres de 
cautiverio de Argentina. Parasitol Latinoam 57: 146-148. doi: 10.4067/ S0717-77122002000300011

18. Miranda H, Fernández W, Ibañez N. 1968. Diphyllobothriasis: Investigación de Diphyllobothrium pacificum (Nybelin, 1931) Margolis, 1956 en Otaria byronia (Sin. Otaria flavescens) y en peces marinos. Arch Per Pat Clin 22: 9-24.

19. Müller GCK, Greinert JA, Silva Filho HH. 2005. Frequência de parasitas intestinais em felinos mantidos em zoológicos. Arq Bras Med Vet Zootec 57: 559-561. doi: 10.1590/S010209352005000400021

20. Müller-Graf CDM. 1995. A coprological survey of parasites of wild lions (Panthera leo) in the Serengeti and the Ngorongoro Crater, Tanzania, East Africa. J Parasitol 81: 812-814.

21. Oliveira T, Moreira N, Eizirik E, Gonçalvez, R, Crawshaw P, Morato RG. 2001. Order Carnivora, Family Felidae (cats). In: Fowler ME, Cubas Z (eds). Biology, medicine, and surgery of South American wild animals. EEUU: Wiley-Blackwell. p 291-300.

22. Pacheco V, Cadenillas $R$, Salas E, Tello C, Zeballos H. 2009. Diversidad y endemismo de los mamíferos en el Perú. Rev Perú Biol 16: 5-32.

23. Patton S, Rabinowitz A, Randolph S, Strawbridge S. 1986. A coprological survey of parasites of wild neotropical felidae. J Parasit 72: 517-520.

24. Patton S, Rabinowitz A. 1994. Parasites of wild felidae in Thailand: a coprological survey. J Wildl Dis 30: 472-475.

25. Pence DB, Tewes ME, Laack LL. 2003. Helminths of the ocelot from southern Texas. J Wildl Dis 39: 683-689. doi: 10.7589/0090-3558-39.3.683
26. Pessutti C, Bodini ME, Fernandes L. 2001. Order Carnivora, Family Canidae (dogs, foxes, maned wolves). In: Fowler ME, Cubas Z (eds). Biology, medicine, and surgery of South American wild animals. EEUU: Wiley-Blackwell. p 279290.

27. Sampaio A, Veloso AL, Silva M. 2001. Order Carnivora, Family Procyonidae (raccoons, kinkajous). In: Fowler ME, Cubas Z (eds). Biology, medicine, and surgery of South American wild animals. EEUU: Wiley-Blackwell. p 317-322.

28. Suzán G, Galindo F, Ceballos G 2000. La importancia del estudio de enfermedades en la conservación de fauna silvestre. Vet Méx 31: 223-228.

29. Tantaleán M, Huiza A. 1994. Sinopsis de los parásitos de peces marinos de la costa peruana. Biotempo 1: 53-101.

30. Tantaleán M, Michaud C. 2005. Huéspedes definitivos de Spirometra mansonoides (Cestoda, Diphyllobothriidae) en Perú. Rev Perú Biol 12: $153-$ 157. doi: $10.15381 / \mathrm{rpb} . v 1211.2370$

31. Tantaleán M, Sánchez L, Gómez L, Huiza A. 2005. Acantocéfalos del Perú. Rev Perú Biol 12: 83-92.

32. Tantaleán M, Mendoza L, Riofrío F. 2007. El zorro andino, Pseudalopes culpaeus, un nuevo huésped para Corynosoma obtuscens (Acanthocephala) en el Perú. Rev Perú Biol 14: 5152. doi: 10.15381/rpb.v14i1.1757

33. Thrusfield M. 1990. Epidemiología veterinaria. España: Ed. Acribia. 339 p.

34. Vieira FM, Luque J, Muniz-Pereira L. 2008. Checklist of helminths parasites in wild carnivore mammals from Brazil. Zootaxa 1721: 1-23. 